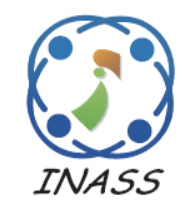

\title{
A Robust Hybrid Watermarking Technique for Securing Medical Image
}

\author{
Imane Assini $^{1 *}$ Abdelmajid Badri $^{1} \quad$ Khadija Safi $^{1} \quad$ Aicha Sahel $^{1} \quad$ Abdennaceur Baghdad $^{1}$ \\ ${ }^{I}$ EEA \& TI Laboratory Faculty of Sciences and Techniques (FSTM), Hassan II University Casablanca, \\ BP 146, Mohammedia, 20650, Morocco \\ * Corresponding author's Email: assini.media@ gmail.com
}

\begin{abstract}
In the aim to contribute to the security of medical image, we present a robust watermarking method which combines discrete wavelet transform (DWT), discrete cosine transform (DCT) and singular value decomposition (SVD). This approach is intended to insert an invisible image watermark in a medical image. The cover medical image is divided up into the third level of DWT coefficients and then is transformed by DCT and SVD. The same procedure is applied to the watermark image. The singular value of watermark is inserted into the singular value of the high-frequency sub bands of the third level DWT of the cover image. However, the insertion of the watermark in these areas makes it possible to reinforce the robustness of the system of watermarking without hindering the quality of the watermarked image. The performance of the proposed method was evaluated in term of invisibility by calculating the Peak Signal to Noise Ratio (PSNR) between the original and the watermarked image and in term of robustness by measuring the normalized correlation coefficient (NC) between the original watermark and the extracted watermark after applying attacks. The experimental results approve that our proposed hybrid algorithm gives an excellent compromise imperceptibility and robustness against several attacks such as Gaussian noise, Saltand-Pepper, Speckle noise, Average filter, Median filter, and Wiener filter compared with existing methods.
\end{abstract}

Keywords: Image processing, Securing, Medical application, Digital watermarking, DWT, DCT, SVD.

\section{Introduction}

Telemedicine is the use of information and communication technologies to supply clinical health care at a distance, it is a well-known application that facilitates the transfer of an enormous amount of medical data. In order to contribute to the security of sharing and transferring of medical images, the digital watermarking has emerged as an alternative and complementary solution to ensure authorized access and content authentication [1].

The system of watermarking should be imperceptible (not affect the visual quality of the cover medical image) and robust (the detection of watermark should be possible even if the image undergoes several attacks). The insertion of the watermark is generally performed in the spatial domain or the frequency domain.
In the spatial domain the insertion is done directly in the values of the pixels of the original image $[2,3]$, it's easy to implement but is fragile against attacks. For example, adding noise or lossy compression can easily degrade the quality of an image or remove the watermark.

Compared to the spatial domain, the insertion in transform domain enhances the performance of watermarking by choosing the pixels that will be more resistant and robust against various attacks such as filtering, noise, and compression. The watermark is inserted by modulating the coefficients of a transform. Among the transforms used in the algorithms of digital images watermarking, we can cite discrete Fourier transform (DFT), discrete cosine transform (DCT), discrete wavelet transform (DWT) and singular value decomposition (SVD). Moreover, the combination of these transformations can increase the efficiency of the watermarking scheme. There are some contributions which propose hybrid methods in the literature like: 
Kumar et al [4] proposed a hybrid method of watermarking based on DWT and SVD.

In this paper, the cover image is divided into the third level of DWT. Afterward, the watermark is inserted into the singular values of HL3 and LH3 sub bands.

In [5], the authors present a robust hybrid technique of watermarking based on DWT, DCT, and SVD. After the decomposition of the cover image into first level DWT, the low-frequency subband of the host image and watermark image are transformed by using DCT and SVD. Then the singular value of the watermark image is integrated into the singular value of the cover image. The watermarked image is obtained by inverse SVD, DCT, and DWT respectively.

In [6], the authors present a new technique of watermarking based on DWT-DCT to insert the watermark into the medical images. The first step consists of decomposing the medical image into four sub-bands, low frequency (LL) and high-frequency image's details (HL, LH, HH). The second step is to apply DCT on HH sub-band and then embeds the watermark. The watermarked image is constructed by inverse DCT and DWT.

In this paper, a robust watermarking technique for medical images based on DWT, DCT, and SVD has been proposed. The strong points of this approach, it is that the insertion of watermark is made into high frequency sub bands $\mathrm{HH}$, what offers a better invisibility, as well as the decomposition DWT up to third level makes it possible to increase the robustness of the system of watermarking against various attacks.

The article is classified as follows: section 2 presents the techniques of watermarking used in our method and describes their advantages. In section 3, the proposed hybrid technique DWT-DCT-SVD has been presented with a description of the steps of watermark embedding and extracting algorithms. Section 4 presents the experimental results obtained using Matlab R2013b and finally the conclusion of work is drawn in Section 5.

\section{Background and theory}

The proposed technique of watermarking medical image was based on DWT-DCT-SVD. One of the advantages of the wavelet transform DWT is that it is based on the characteristics of the human visual system. This allows us to use the watermark in the regions where the HVS is less sensitive like high-resolution detail bands. The insertion of the watermark in these regions makes it possible to increase the robustness without affecting the quality of the image.

The DCT reduces the spatial correlation between the pixels of an image and it offers a good robustness to attacks such as the adjustment of contrast, filtering, and compression. And finally, The SVD stores the maximum energy of the image in a minimum of singular values. The main advantage of this method is that the singular values (SV) are very stable.

\subsection{Discrete wavelet transform (DWT)}

DWT is a new technique which is used to represent an image in a new time and frequency scale in recent years [1]. The transform is based on small waves called wavelet of varying frequency.

Applying wavelet transform [7] on twodimensional images divides the image into four subbands: a lower resolution approximation image (LL), horizontal (HL), vertical (LH) and diagonal (HH) detail components. The maximum energy of an image is concentrated in low-frequency sub-band (LL) whereas high-frequency components sub-band ( $\mathrm{HL}, \mathrm{LH}$, and $\mathrm{HH}$ ) correspond to edges and textures.

\subsection{Discrete cosine transform (DCT)}

Discrete Cosine Transform (DCT) [8] is the most popular transform function used in signal processing. It transforms a signal from spatial domain to frequency domain. The insertion of the watermark is done in the middle frequency.

The discrete cosine transform of an image $x$ of dimensions $N \times N$ is computed according to Eq. (1):

$\operatorname{DCT}(m, n)=$

$$
\left.\begin{array}{l}
\frac{1}{\sqrt{2 N}} c(m) \cdot c(n) \sum_{k=0}^{N-1} \sum_{l=0}^{N-1} x(k, l) \cos \left(\frac{(2 k+1) m \pi}{2 N}\right) \cos \left(\frac{(2 l+1) n \pi}{2 N}\right) \\
C(m), C(n)= \\
\left\{\frac{1}{\sqrt{N}}, m, n=0 ; \sqrt{\frac{2}{N}}, m, n=1 \text { up to } N-1\right.
\end{array}\right\}
$$

\subsection{Singular value decomposition (SVD)}

The singular value decomposition [9] of a matrix I is the factorization of the form:

$$
I=U S V^{T}
$$




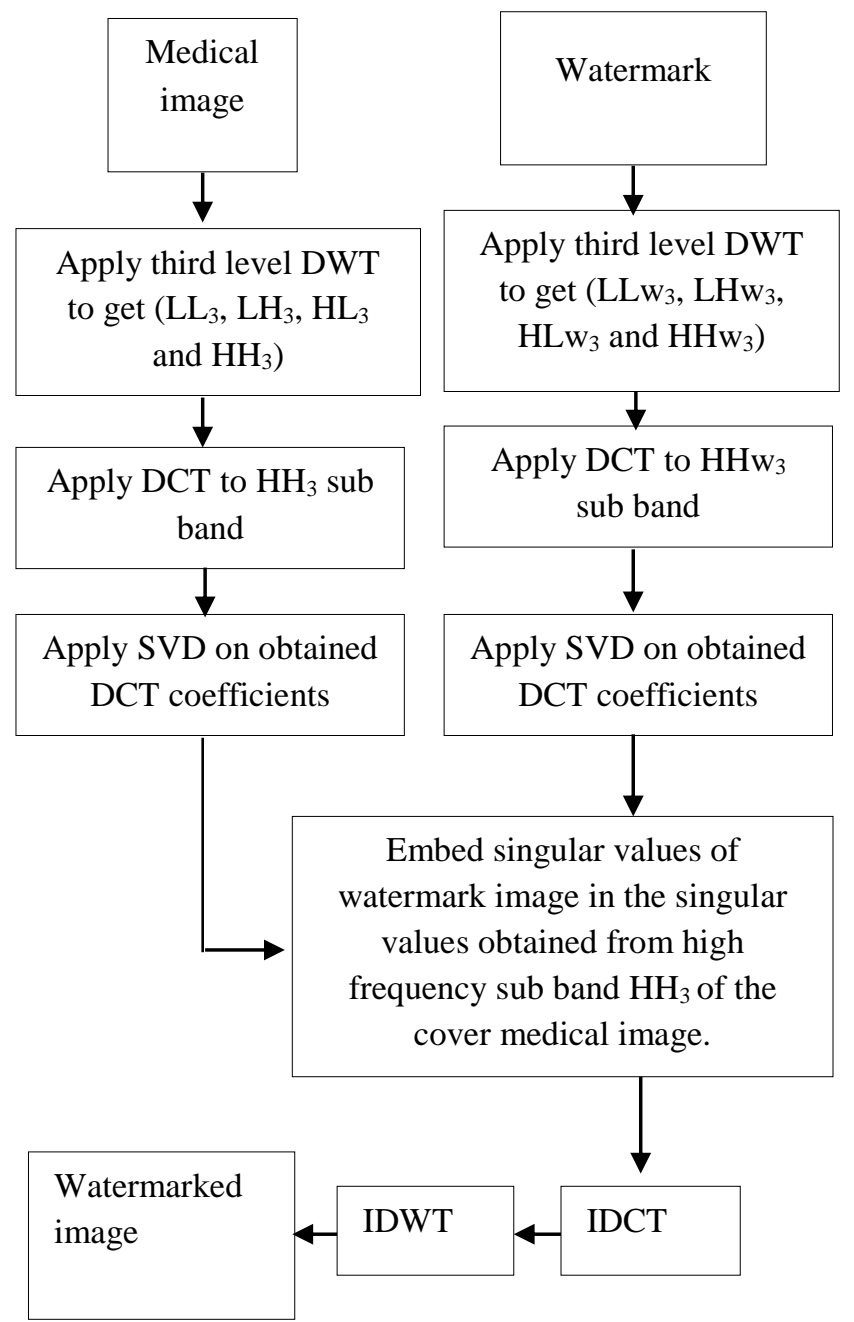

Figure.1 Watermark embedding process using DWTDCT-SVD

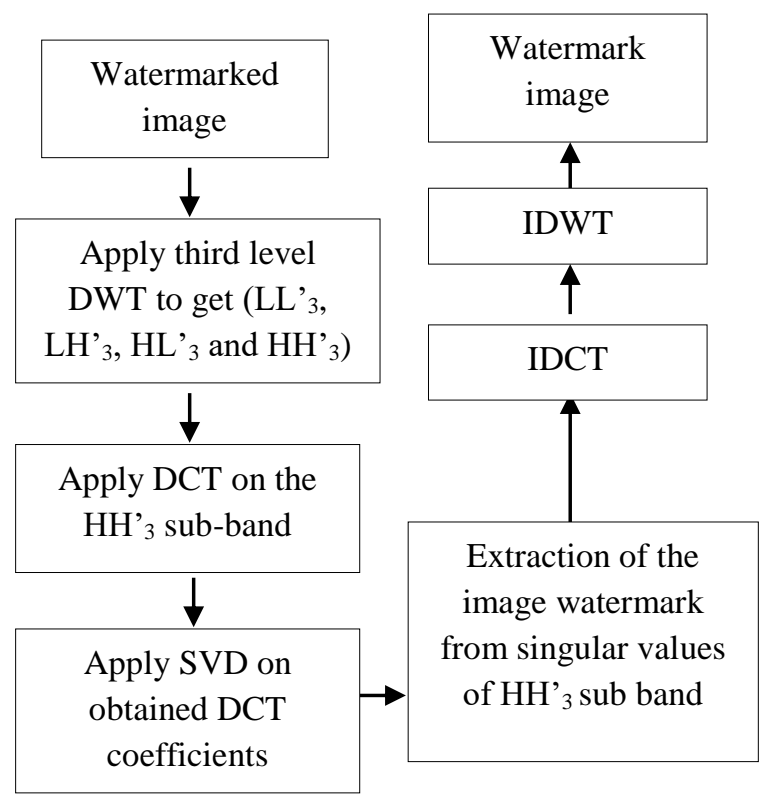

Figure.2 Watermark extraction process using DWT-DCTSVD
Where $\mathrm{U}$ and $\mathrm{V}$ are orthogonal matrices of size $N \mathrm{x} N$ and $S$ is a diagonal matrix which comprises the singular values $(s 1, s 2, \ldots \ldots, s N)$ of the matrix I.

\section{Proposed hybrid technique}

The aim of this article is to present and analyze a robust method of watermarking based on a combination of three techniques: DWT, DCT, and SVD.

The ultimate goal of this proposed method is to increase the robustness of the system in terms of quality and confidentiality. Fig. 1 and Fig. 2 illustrates the watermark embedding and extracting process respectively.

\subsection{Watermark embedding algorithm}

1. Apply the third level DWT transform using Haar wavelet on cover medical image and watermark image to get $\left(\mathrm{LL}_{3}, \mathrm{HL}_{3}, \mathrm{LH}_{3}\right.$, $\left.\mathrm{HH}_{3}\right)$ and $\left(\mathrm{LLw}_{3}, \mathrm{HLw}_{3}, \mathrm{LHw}_{3}, \mathrm{HHw}_{3}\right)$ subbands respectively.

2. Apply DCT to the selected sub-bands $\left(H_{3}, H H_{W 3}\right)$ and then apply SVD to obtain Eq. (3) and Eq. (4):

$$
\begin{aligned}
& I=U_{H H 3} \times S_{H H 3} \times V_{H H 3^{T}} \\
& W=U_{W} \times S_{W} \times V_{W^{T}}
\end{aligned}
$$

3. Modify the singular values of HH3 subband of the cover image with the singular values of $H_{W}$ sub-band of the watermark image:

$S_{I W}=S_{H H 3}+\left(\alpha \times S_{W}\right)$

Such as ( $\alpha$ : scaling factor)

4. Apply inverse DCT and inverse DWT to produce the watermarked medical image.

\subsection{Watermark extracting algorithm}

1. Apply the third level DWT transform on the watermarked image to get LL'3, HL'3, LH'3 and HH'3 sub-bands.

2. Apply DCT and then SVD to the selected sub-band $\mathrm{HH}_{3}$ :

$$
I_{\text {wat }}=U_{H^{\prime} 3} \times S_{H^{\prime} 3} \times V_{H H^{\prime} 3}
$$


3. Obtain singular values of the watermark from singular values of high-frequency subbands $\left(\mathrm{HH}_{3}, \mathrm{HH}_{3}\right)$ of watermarked image and cover image respectively by Eq. (7):

$$
S_{e W}=\frac{\left(S_{H H / 3}-S_{H H 3}\right)}{\alpha}
$$

4. Obtain extracted watermark by applying inverse singular value decomposition by using following Eq. (8):

$$
W^{\prime}=U_{W} \times S_{e w} \times V_{W}
$$

5. Apply inverse DCT and inverse DWT to obtain final extracted watermark.

\section{Experimental results and analysis}

The proposed DWT-DCT-SVD based scheme was implemented in Matlab R2013b.

Various experiments are performed on two cover medical images 'Megacolon' and 'Abdominal' of size " $512 \times 512$ " and 'girl face', 'fingerprint' of size " $512 \times 512$ " taken as watermark images shown in Fig. 3 and 4.The performance of the proposed watermarking approach is evaluated in terms of imperceptibility and robustness against various attacks.

The Peak Signal to Noise Ratio (PSNR The Peak Signal to Noise Ratio (PSNR): measures the similarity between the original image and the watermarked image [10]:

$$
P S N R=10 \log \left[\left((255)^{2}\right) / M S E\right]
$$

Where MSE [10] represents the mean square error to measure the perceptual distance between watermarked and original image.

MSE can be defined as [11]:

$$
M S E=\frac{1}{M N} \sum_{i=1}^{M} \sum_{j=1}^{N}\left[I(i, j)-I^{\prime}(i, j)\right]^{2}
$$

Where I and I' are the original image and the watermarked image of size $\mathrm{M}$ x $\mathrm{N}$ respectively.

However, robustness is measured by the normalized correlation coefficient (NC) [12]:

$$
N C=\frac{\sum_{i} \sum_{j}(\boldsymbol{W}(i, j) * \boldsymbol{W} \prime(i, j))}{\sqrt{\left(\sum_{i} \sum_{j}\left(\boldsymbol{W}_{(i, j)}\right)^{2}\right)\left(\sum_{i} \sum_{j}\left(\boldsymbol{W}^{\prime}(i, j)\right)^{2}\right)}}
$$

Where $\mathrm{W}(i, j)$ and $\mathrm{W}^{\prime}(i, j)$ are the pixel intensity values at coordinates $(i, j)$ of original and extracted watermark respectively.

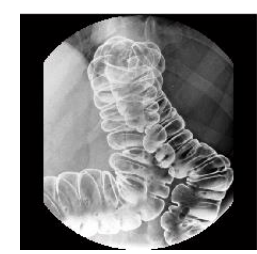

(a)

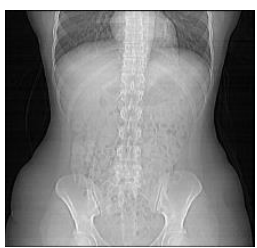

(b)
Figure. 3 Cover medical images: (a) megacolon and (b) abdominal

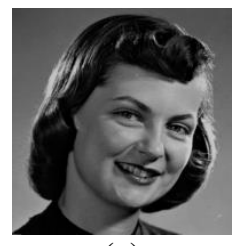

(a)

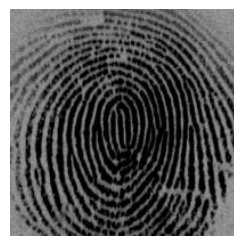

(b)
Figure.4 Watermark images: (a) girl face and (b) fingerprint

The imperceptibility of a watermark is measured by calculating the PSNR between the original image and the watermarked image. Fig.5 shows the application of our method DWT-DCT-SVD, there is no visual difference between the cover medical images and watermarked images.

In Table 1 and Table 2, the performance of the proposed method for two cover medical images 'Megacolon' and 'Abdominal' against watermark images 'Girl face' and 'Fingerprint' had been evaluated without any noise attacks.

In the case where the original image is 'Megacolon', the maximum PSNR values obtained are $58.03 \mathrm{~dB}$ and $51.78 \mathrm{~dB}$ and minimum PSNR values are $39.46 \mathrm{~dB}$ and $33.09 \mathrm{~dB}$ against inserted watermarks 'Girl face' and 'Fingerprint' at scaling factor $(\alpha)=0.1$ and 0.9 respectively.

In the other case where the original image is 'Abdominal', the maximum PSNR values obtained are $57.80 \mathrm{~dB}$ and $51.26 \mathrm{~dB}$ and minimum PSNR values are $39.02 \mathrm{~dB}$ and $32.56 \mathrm{~dB}$ against inserted watermarks 'Girl face' and 'Fingerprint' at scaling factor $(\alpha)=0.1$ and 0.9 respectively.

However, the $\mathrm{NC}$ values in all scaling factor are 1 in the both cases.

According to the interpretation of the results shown in tables 1 and 2 , we conclude that the insertion of the watermark by the proposed technique gives good invisibility. 


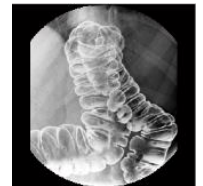

(a)

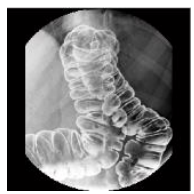

(d)

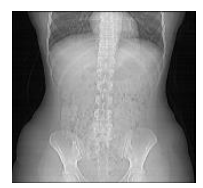

(g)

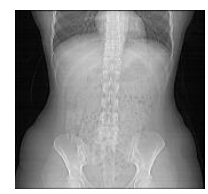

(j)

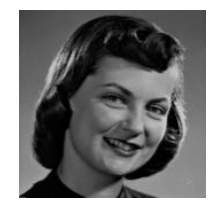

(b)

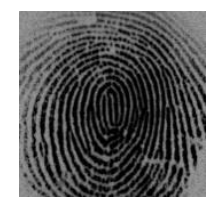

(e)

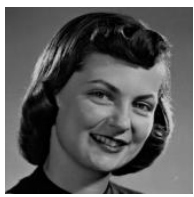

(h)

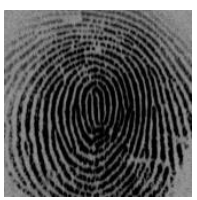

(k)

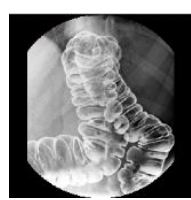

(c)

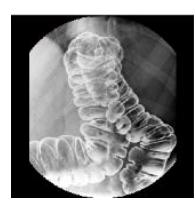

(f)

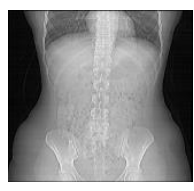

(i)

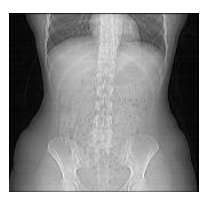

(1)
Figure.5 (a, d, g, j) Cover medical images, (b, e, h, k) Watermark images, and (c, f, i, l) Watermarked images

Table1. PSNR, NC performance of the proposed method at different gain factor for cover medical image 'megacolon'

\begin{tabular}{ccccc}
\hline $\begin{array}{c}\text { Gain } \\
\text { factor } \\
(\boldsymbol{\alpha})\end{array}$ & \multicolumn{2}{c}{$\begin{array}{c}\text { Watermark } \\
\text { (Girl face) }\end{array}$} & \multicolumn{2}{c}{$\begin{array}{c}\text { Watermark } \\
\text { (Fingerprint) }\end{array}$} \\
\cline { 2 - 5 } & PSNR & NC & PSNR & NC \\
\hline 0.1 & 58.03 & 1 & 51.78 & 1 \\
0.2 & 52.24 & 1 & 45.90 & 1 \\
0.3 & 48.82 & 1 & 42.40 & 1 \\
0.4 & 46.34 & 1 & 39.93 & 1 \\
0.5 & 44.42 & 1 & 38.06 & 1 \\
0.6 & 42.88 & 1 & 36.51 & 1 \\
0.7 & 41.62 & 1 & 35.20 & 1 \\
0.8 & 40.47 & 1 & 34.07 & 1 \\
0.9 & 39.46 & 1 & 33.09 & 1 \\
\hline
\end{tabular}

Table2. PSNR, NC performance of the proposed method at different gain factor for cover medical image 'abdominal'.

\begin{tabular}{ccccc}
\hline $\begin{array}{c}\text { Gain } \\
\text { factor } \\
(\boldsymbol{\alpha})\end{array}$ & \multicolumn{2}{c}{$\begin{array}{c}\text { Watermark } \\
\text { (Girl face) }\end{array}$} & \multicolumn{2}{c}{$\begin{array}{c}\text { Watermark } \\
\text { (Fingerprint) }\end{array}$} \\
\cline { 2 - 5 } & PSNR & NC & PSNR & NC \\
\hline 0.1 & 57.80 & 1 & 51.26 & 1 \\
0.2 & 51.76 & 1 & 45.42 & 1 \\
0.3 & 48.40 & 1 & 41.98 & 1 \\
0.4 & 45.86 & 1 & 39.52 & 1 \\
0.5 & 44.03 & 1 & 37.62 & 1 \\
0.6 & 42.50 & 1 & 36.03 & 1 \\
0.7 & 41.13 & 1 & 34.71 & 1 \\
0.8 & 40.02 & 1 & 33.57 & 1 \\
0.9 & 39.02 & 1 & 32.56 & 1 \\
\hline
\end{tabular}

To test and check the robustness of our watermarking algorithm, there must be a strong correlation between the watermark and the extracted watermark after various attacks. In general, an NC value is acceptable if it is 0.75 or higher [13].

The robustness of the proposed watermarking scheme has been demonstrated by applying various noise attacks on the watermarked image such as Gaussian noise, Salt \& pepper, Speckle noise and filtering attacks such as Average filter, Median filter and Wiener filter shown in Table 3, 4, 5 and 6.

In Table 3 and 4, the NC values of extracted watermarks 'Girl face' and 'Finger print' of test medical images have been evaluated for $\mathrm{HH}$ band at scaling factor $(\alpha)=0.8$ respectively, the results show that the proposed scheme has high degree of robustness than results reported by [5].

At high density (0.08) of Gaussian noise, Salt \& pepper and Speckle noise, the efficiency of the proposed method gives maximum NC of 0.9997 , $0.9998,0.9999$ compared to $0.9523,0.9468,0.9458$ obtained by [5]. And the efficiency of the proposed method against Average filter $[5,5]$ gives maximum $\mathrm{NC}$ of 0.9976 compared to 0.9909 obtained by [5].

The drawbacks of [5] compared to our contribution is that the insertion of the watermark was made in the low frequency bands (LL) of the first level DWT of the original image. However, in our approach, the watermark was inserted in the high frequency sub-bands of the third level of DWT which offers a better robustness.

The robustness of the proposed method against Gaussian noise (0.1), Salt and pepper (1.0), Speckle noise $(0.1)$ and Average filter [13, 13] has been compared with [13], shown in Table 5.

In Table 6, the maximum $\mathrm{NC}$ values at scaling factor $(\alpha)=0.8$ with proposed method has been obtained as 1 for Gaussian noise (0.01) against $0.9830,0.9582,0.8152$ obtained by [14], [15] and [16] respectively, 1 for Salt and pepper noise 0.01 against $0.9972,0.9888$ and 0.8863 obtained by [14], [15] and [16] respectively, and finally 1 for speckle noise 0.01 against 0.9868 and 0.7382 obtained by [15] and [16] respectively.

The performance of the proposed watermarking scheme which based on the advantages of combination DWT-DCT-SVD and the insertion in the high frequency sub bands gives very good results compared with other reported techniques. 
Table 3. Normalized correlation coefficient of extracted watermark 'Girl face' from test medical images at scaling factor $=0.8$

\begin{tabular}{lccc}
\hline Attacks & $\begin{array}{c}\text { NC values (Proposed } \\
\text { method using medical } \\
\text { image 'Megacolon') }\end{array}$ & $\begin{array}{c}\text { NC values (Proposed } \\
\text { method using medical } \\
\text { image 'Abdominal') }\end{array}$ & $\begin{array}{c}\text { NC values of [5] } \\
\text { method }\end{array}$ \\
\hline Gaussian noise with mean=0, Var-0.01 & 0.9999 & 0.9999 & 0.9872 \\
Gaussian noise with mean=0, Var-0.02 & 0.9998 & 0.9996 & 0.9841 \\
Gaussian noise with mean=0, Var-0.03 & 0.9998 & 0.9992 & 0.9803 \\
Gaussian noise with mean=0, Var-0.04 & 0.9996 & 0.9989 & 0.9760 \\
Gaussian noise with mean=0, Var-0.05 & 0.9995 & 0.9983 & 0.9709 \\
Gaussian noise with mean=0, Var-0.06 & 0.9994 & 0.9975 & 0.9649 \\
Gaussian noise with mean=0, Var-0.07 & 0.9991 & 0.9973 & 0.9587 \\
Gaussian noise with mean=0, Var-0.08 & 0.9988 & 0.9966 & 0.9523 \\
Salt \& Pepper with (Density=0.01) & 1 & 0.9999 & 0.9962 \\
Salt \& Pepper with (Density=0.02) & 0.9999 & 0.9998 & 0.9917 \\
Salt \& Pepper with (Density=0.03) & 0.9998 & 0.9997 & 0.9869 \\
Salt \& Pepper with (Density=0.04) & 0.9998 & 0.9995 & 0.9799 \\
Salt \& Pepper with (Density=0.05) & 0.9997 & 0.9992 & 0.9729 \\
Salt \& Pepper with (Density=0.06) & 0.9996 & 0.9989 & 0.9641 \\
Salt \& Pepper with (Density=0.07) & 0.9995 & 0.9987 & 0.9551 \\
Salt \& Pepper with (Density=0.08) & 0.9994 & 0.9983 & 0.9468 \\
Speckle noise with (Density=0.01) & 1 & 1 & 0.9981 \\
Speckle noise with (Density=0.02) & 1 & 1 & 0.9906 \\
Speckle noise with (Density=0.03) & 0.9999 & 1 & 0.9849 \\
Speckle noise with (Density=0.04) & 0.9999 & 0.9786 \\
Speckle noise with (Density=0.05) & 0.9999 & 0.9999 & 0.9718 \\
Speckle noise with (Density=0.06) & 0.9999 & 0.9998 & 0.9631 \\
Speckle noise with (Density=0.07) & 0.9999 & 0.9998 & 0.9540 \\
Speckle noise with (Density=0.08) & 0.9999 & 0.9998 & 0.9458 \\
AverageFilter[5, 5] & 0.9960 & 0.9996 & 0.9909 \\
\hline
\end{tabular}

Table 4.Normalized correlation coefficient of extracted watermark 'Fingerprint' from test medical images at scaling factor $=0.8$

\begin{tabular}{lccc}
\hline Attacks & $\begin{array}{c}\text { NC values (Proposed } \\
\text { method using medical image } \\
\text { 'Megacolon') }\end{array}$ & $\begin{array}{c}\text { NC values (Proposed } \\
\text { method using medical } \\
\text { image 'Abdominal') }\end{array}$ & $\begin{array}{c}\text { NC values of [5] } \\
\text { method }\end{array}$ \\
\hline Gaussian noise with mean=0, Var-0.01 & 0.9998 & 1 & 0.9872 \\
Gaussian noise with mean=0, Var-0.02 & 0.9998 & 0.9999 & 0.9841 \\
Gaussian noise with mean=0, Var-0.03 & 0.9998 & 0.9999 & 0.9803 \\
Gaussian noise with mean=0, Var-0.04 & 0.9998 & 0.9997 & 0.9760 \\
Gaussian noise with mean=0, Var-0.05 & 0.9998 & 0.9997 & 0.9709 \\
Gaussian noise with mean=0, Var-0.06 & 0.9998 & 0.9995 & 0.9649 \\
Gaussian noise with mean=0, Var-0.07 & 0.9997 & 0.9995 & 0.9587 \\
Gaussian noise with mean=0, Var-0.08 & 0.9997 & 0.9992 & 0.9523 \\
Salt \& Pepper with (Density=0.01) & 0.9998 & 1 & 0.9962 \\
Salt \& Pepper with (Density=0.02) & 0.9998 & 1 & 0.9917 \\
Salt \& Pepper with (Density=0.03) & 0.9998 & 0.9999 & 0.9869 \\
Salt \& Pepper with (Density=0.04) & 0.9998 & 0.9999 & 0.9799 \\
Salt \& Pepper with (Density=0.05) & 0.9998 & 0.9998 & 0.9729 \\
Salt \& Pepper with (Density=0.06) & 0.9998 & 0.9997 & 0.9641 \\
Salt \& Pepper with (Density=0.07) & 0.9998 & 0.9996 & 0.9551 \\
Salt \& Pepper with (Density=0.08) & 0.9998 & 0.9995 & 0.9468 \\
Speckle noise with (Density=0.01) & 0.9998 & 1 & 0.9981 \\
Speckle noise with (Density=0.02) & 0.9998 & 1 & 0.9906 \\
Speckle noise with (Density=0.03) & 0.9998 & 1 & 0.9849 \\
Speckle noise with (Density=0.04) & 0.9998 & 0.9999 & 0.9786 \\
Speckle noise with (Density=0.05) & 0.9998 & 0.9999 & 0.9718 \\
Speckle noise with (Density=0.06) & 0.9998 & 0.9999 & 0.9631 \\
Speckle noise with (Density=0.07) & 0.9998 & 0.9999 & 0.9540 \\
Speckle noise with (Density=0.08) & 0.9998 & 0.9999 & 0.9458 \\
AverageFilter[5, 5] & 0.9919 & 0.9940 & 0.9909 \\
\hline
\end{tabular}


Table 5. Performance of proposed method against Gaussian noise (0.1), Salt \& pepper (1), Speckle noise (0.1) and Average Filter $[13,13]$

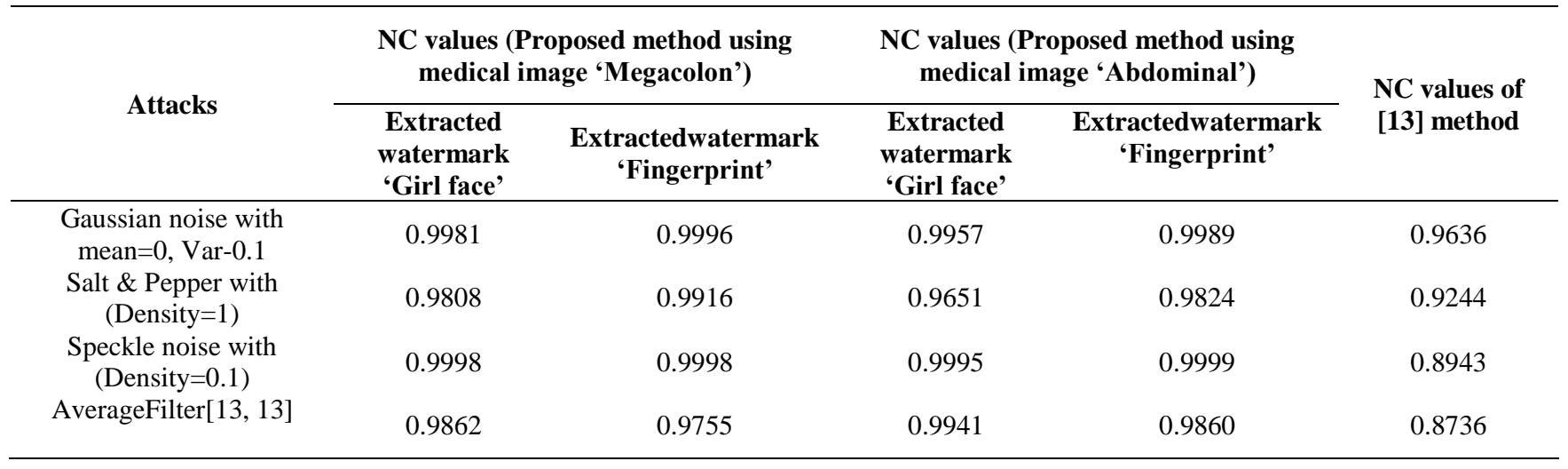

Table 6. Comparison of NC values with existing methods at gain $=0.8$

\begin{tabular}{|c|c|c|c|c|}
\hline \multirow{2}{*}{ Attacks } & \multicolumn{3}{|c|}{ Existing Schemes } & \multirow{2}{*}{$\begin{array}{c}\text { Proposed } \\
\text { scheme }\end{array}$} \\
\hline & [14] & [15] & [16] & \\
\hline Gaussian noisemean $=0$, Var -0.01 & 0.9830 & 0.9582 & 0.8152 & 1 \\
\hline Gaussian noisemean $=0$, Var -0.02 & Not shown & 0.9457 & Not shown & 0.9999 \\
\hline Gaussian noisemean $=0$, Var -0.05 & 0.9743 & Not shown & Not shown & 0.9998 \\
\hline Gaussian noisemean $=0$, Var- 0.005 & Not shown & Not shown & 0.8531 & 1 \\
\hline Salt \& Pepper noise (Density=0.01) & 0.9972 & 0.9888 & 0.8863 & 1 \\
\hline Salt \& Pepper noise (Density=0.05) & 0.9955 & Not shown & Not shown & 0.9998 \\
\hline Salt \& Pepper noise (Density=0.003) & Not shown & Not shown & 0.9576 & 1 \\
\hline Speckle noise $($ Density $=0.01)$ & Not shown & 0.9868 & 0.7382 & 1 \\
\hline Speckle noise $($ Density $=0.05$ ) & 0.9955 & Not shown & Not shown & 0.9999 \\
\hline Averagefilter & 0.9975 & Not shown & 0.9736 & 0.9996 \\
\hline Medianfilter $[3,3]$ & 0.9988 & Not shown & 0.7774 & 0.9995 \\
\hline Wienerfilter $[3,3]$ & 0.9989 & Not shown & 0.7890 & 0.9997 \\
\hline
\end{tabular}

\section{Conclusion}

In this paper, a hybrid watermarking technique DWT-DCT-SVD has been presented to ensure the copyright protection and security of medical image.

The proposed watermarking algorithm combines the advantages of DWT, DCT, and SVD. The DWT provides better identification of appropriate data based on the characteristics of the human visual system HVS. The DCT gives good perceptual invisibility and robustness. And finally, the main advantage of SVD is that the singular values are very stable, when small information (perturbations) is added into an image, their singular values do not change significantly.

Many tests have allowed highlighting our proposed technique, it has the advantage to insert a large amount of data (watermark of size 512x512) without impacting the quality of the image, as well as its robustness against various attacks.

The simulation results in term of invisibility gives a maximum PSNR value $=58.03 \mathrm{~dB}$ at scaling factor 0.1 and in term of robustness gives a maximum $\mathrm{NC}$ value $=1$ against noise attacks such as Gaussian noise, Salt \& pepper and Speckle noise, also an excellent $\mathrm{NC}$ values against filtering attacks like Average filter, Median filter and Wiener filter compared to other reported techniques. These results approve that our contribution is able to give a very good compromise imperceptibility and robustness.

In the future research, we will try to enhance the performance of our algorithm in terms of robustness, capacity and imperceptibility and to further research on techniques of watermarking for medical applications.

\section{References}

[1] I. Assini, A. Badri, and K. Safi "Adaptation of Different Techniques on Digital Image Watermarking in Medical Domain: A review", International Journal of Advanced Research in Computer Science and Software Engineering (IJARCSSE), Vol.5, No.12, pp.5-9, 2015.

[2] M. D. Swanson, M. Kobayashi, and A. H. Tewfik, "Multimedia data-embedding and watermarking technologies", In: Proc. of the IEEE, Vol. 86, No.6, pp. 1064-1087, 1998.

[3] W. Bender, D. Gruhl, N. Morimoto, and A. Lu, "Techniques for Data Hiding", IBM Systems Journal, Vol.35, No.3.4, pp.313-336, 1996. 
[4] S. Kumar, A. K. Saini, and P. Kumar, "SVD based robust digital image watermarking using discrete wavelet transform", Int. J. of Comp. Appl, Vol. 45, No. 10, pp. 7-11, 2012

[5] A. K. Singh, M. Dave, and A. Mohan, "Hybrid Technique for Robust and Imperceptible Image Watermarking in DWT-DCT-SVD Domain", The National Academy Science Letters, Vol.37, No.4, pp. 351-358, 2014.

[6] A. Mehto and N. Mehra, "Adaptive Lossless Medical Image Watermarking Algorithm Based on DCT \& DWT", Procedia Computer Science, Vol.78, pp. 88-94, 2016.

[7] H. B. Kekre, T. Sarode, and S. Natu, "Performance Comparison of DCT and walsh Transforms for watermarking using DWTSVD”, International Journal of Advanced Computer Science and Application, Vol.4, No.2, 2013.

[8] C. Dong, H. Zhang, J. Li, and Y. Chen, "Robust Zero-Watermarking for Medical Image Based on DCT", In: Proc. of the 6th International Conference on Computer Sciences and Convergence Information Technology, pp.900904, 2011.

[9] C. C. Chang, P. Tsai, and C. C. Lin, "SVDbased digital image watermarking scheme", Pattern Recognition Letters, Vol.26, No.10, pp.1577-1586, 2005.

[10] S. Mchta, R. Nallusamy, R. V. Marawar, and B. Prabhakaran, "A Study of DWT and SVD based watermarking Algorithms for patient Privacy in Medical Images", In: Proc. of the 2013 IEEE International Conference on Healthcare Informatics, pp.287-296, 2013.

[11] K. chaitanya, S. Reddy, and G. Rao, "Digital Color Image Watermarking In RGB Planes Using DWT-DCT-SVD coefficients", International Journal of Computer Science and Information Technologies, Vol.5, No.2, pp.2413-2417, 2014.

[12] N. J. Harish, B. B. S. Kumar, and A. Kusagur "Hybrid robust watermarking techniques based on DWT, DCT, and SVD", International Journal of Advanced Electrical and Electronics Engineering, Vol.2, No.5, pp.137-143, 2013.

[13] D. Singh and S. K. Singh "DWT-SVD and DCT based robust and blind watermarking scheme for copyright protection", Multimedia Tools and Applications, Vol.76, No.11, pp.13001-13024, 2017.

[14] S. Singh, R. Singh, A. K. Singh, and T. J. Siddiqui, "SVD- DCT Based medical image watermarking in NSCT domain", In: Quantum Computing: An Environment for Intelligent Large Scale Real Application, Springer, Cham, pp. 467-488, 2018.

[15] A. Sharma, A. K. Singh, and P. Kumar, "Combining Haar Wavelet and Karhunen-Loeve Transform for Robust and Imperceptible Data Hiding Using Digital Images", Journal of Intelligent Systems, Vol. 27, No 1, pp. 91-103, 2018.

[16] S. Roy and A. K. Pal, "A robust blind hybrid image watermarking scheme in RDWT-DCT domain using Arnold scrambling", Multimedia Tools and Applications, Vol. 76, No 3, pp. 3577-3616, 2017. 